\title{
An assessment of WISC-IIIUK on children with HIV infection
}

Journal of Health Psychology

$1-12$

(C) The Author(s) 2015

Reprints and permissions:

sagepub.co.uk/journalsPermissions.nav DOI: $10.1177 / 13591053155771 / 8$

hpq.sagepub.com

@AGE

\author{
Anu Nikitha James' and Miriam Ittyerah ${ }^{2}$
}

\begin{abstract}
The Wechsler Intelligence Scale for Children - Third Edition UK test was administered to groups of children between the ages of 6 and 12 years with vertically transmitted HIV infection $(n=70)$ and a control group who were not infected by the virus $(n=70)$. The study was conducted in India. The two groups were matched for general verbal abilities, age and gender. The children were assessed for Verbal IQ, Performance IQ and Full-Scale IQ. The Verbal Comprehension Index, Perceptual Organization Index and Freedom from Distractibility Index were also obtained. A three-factor analysis of variance disclosed that school-age children with vertically transmitted HIV infection notched below in the areas of Verbal IQ, Performance IQ, Full-Scale IQ, Verbal Comprehension Index, Perceptual Organization Index and Freedom from Distractibility Index when collated with normal uninfected cohorts. Findings are discussed in the light of both theoretical and clinical implications.
\end{abstract}

\section{Keywords}

HIV infection, intellectual ability, neuropsychological effects, Wechsler Intelligence Scale for Children Third Edition UK

\section{Introduction}

Most children suffer from HIV disease unknowingly, involuntarily or due to circumstances. Children with HIV have been infected through mother-to-infant (vertical transmission) or through contaminated blood or blood products (horizontal transmission) and sometimes with no specific risk factors. The term vertical transmission refers to infection of children from the mother, which happens either in uterus, during the birth process, or through breastfeeding.

According to the Global Action Programme (GAP) report (UNAIDS, 2014), an estimated 3.2 million children in 2013 were living with HIV infection worldwide, most of these children having acquired it through vertical transmission. The World Health Organization (WHO)/Joint
United Nations Programme for HIV/AIDS (UNAIDS)/United Nations Children's Fund (UNICEF) (2011) quoted that an estimated 390,000 children became newly infected with HIV in 2010. Most children live with HIV/ AIDS - almost 9 in 10 - live in sub-Saharan Africa, the region of the world where AIDS has taken its greatest toll.

\footnotetext{
'Bangalore University, India

Institute for Communicative and Cognitive

Neurosciences, India
}

\section{Corresponding author:}

Miriam Ittyerah, Institute for Communicative and Cognitive Neurosciences, Shoranur 679523, India. Email: miriamittyerah7@yahoo.co.in 
India has the third largest number of people in the world living with HIV/AIDS, totalling 2.3 million adults and 115,000 children. In most recent reports, the National AIDS Control Organization (NACO) estimates that about 57,000 children are infected each year with HIV/AIDS; however, 64,000 children living with HIV/AIDS are registered and 22,837 are on antiretroviral therapy (ART), and the high prevalence states are concentrated in the south of India, namely, Tamil Nadu, Andhra Pradesh, Karnataka, Maharashtra and the north-eastern states of Nagaland and Manipur (United States Agency for International Development (USAID, 2012).

The detrimental effects of children suffering from HIV infection have impeded normal development. Children with HIV infection have displayed delays in cognition and performance as compared to their normal cohorts (Mellins and Ehrhardt, 1994; Nozyce et al., 1994). The frequency and spectrum of neurologic impairment are greater in children than those reported for adults. In a study conducted by Pearson et al. (2000), 722 ART-naive children with symptomatic HIV infection were assessed at study entry and at later intervals. Assessments included neuro-developmental testing, neuroradiologic imaging and neurological examination of motor function. CD4 cell count and plasma RNA viral load were also measured (Centers for Disease Control, 1994). The results showed that children with the lowest neuropsychological functioning (IQ $<70)$ at baseline had the highest risk of later HIV disease progression (56\%), compared with those with borderline/ low (IQ=70-89) functioning $(26 \%)$ or with average or above (IQ>90) functioning (18\%). Motor dysfunction (especially reduced muscle mass) at entry also predicted disease progression. These findings have important implications for predicting long-term outcomes (e.g. longevity) in paediatric patients. The main neurological condition related to HIV infection in childhood is HIV-associated progressive encephalopathy, which may be the initial presenting condition for AIDS in 18 per cent of cases, affecting 30 per cent to 60 per cent of seropositive infants, children and adolescents at any time of their disease. HIV-associated progressive encephalopathy causes neuropsychological deficits involving a wide variety of domains, such as speed and language, memory, learning, information processing and motor functioning. They may negatively affect children's normal development and school achievement (Millana-Cuevas et al., 2007).

Epidemiological research has shown that there are critical differences in disease progression between children and adults. Largely due to the lower efficiency of a child's immature (but developing) immune system, children experience a much more rapid disease progression and a much shorter duration of each stage of HIV disease. In the absence of any intervention, the majority of perinatally HIV-infected children develop HIV-related symptoms by 6 months of age (Spira et al., 1999). Studies in resource-constrained countries indicate that the risk of death in untreated HIV-infected infants is 45 per cent at 2 years and 62 per cent at 5 years (Spira et al., 1999). Delayed diagnosis of HIV infection, poor nutrition and high levels of severe bacterial, respiratory and gastrointestinal tract infections are likely reasons for the higher early mortality (Spira et al., 1999). The developmental delays in HIV-infected children can, however, also be caused by adverse rearing environment (Brown et al., 2000).

Improvements in diagnosis and treatment have extended life spans for children infected with HIV/AIDS early in the epidemic. The introduction of ART has transformed the HIV/ AIDS epidemic worldwide. In every setting where ART has been used, death rates have dropped, as well as the incidence of hospitalizations and incidence of opportunistic infections in children (Fassinou et al., 2004; Gortmaker et al., 2001; Puthanakit et al., 2005). The primary goal of ART is to prevent clinical complications of HIV and to prolong the survival of HIVinfected children (Fassinou et al., 2004; Gortmaker et al., 2001; Puthanakit et al., 2005). However, among children and adolescents, a combination of therapies and essential drugs brings down the intermediate-term risk of 
death, HIV-related disease progression and neurological impairments (Greenfield et al., 2013). Hence, researches on understanding their intellectual functioning and other abilities will compliment coping with their present lifestyle.

HIV is the ninth leading cause of death in children (Wolters et al., 1995). In India, studies relating to the cognitive development of children with HIV infection are yet limited in number and less well developed than in adult HIV. This research is focussed on the intellectual functioning in children with HIV infection since there is little comprehensive research. The study chose school-age children, as school-age children represent an important age group because, with improvements in ART, children live longer and healthier. Since HIV-infected children are now surviving well in their school age and adolescence, it is important to understand their intellectual functioning to plan for their educational and mental health needs. We chose to measure a broad range of cognitive skills with a comprehensive battery of tests (Wechsler Intelligence Scale for Children Third Edition UK (WISC-IIIUK) selected for their utility to help children to be successful at school and thereby improve their quality of life.

\section{Method}

\section{Participants}

The sample contained two groups, one with 70 children who had vertically transmitted HIV infection ranging between the ages of 6 and 12.11 years ( 35 males and 35 females) and the other a control group of 70 children $(35$ males and 35 females) who were not infected by the virus. The study was conducted in India. The primary language used to administer the test was English. The two groups were matched for age, gender and ability to communicate in English language (samples were selected from schools where English was the primary language for communication). At each age level, there were five male participants and five female participants in both the experimental and control groups that served to identify the gender difference in intellectual functioning. Children with any physical or mental disability were excluded from the study.

\section{Selection criteria}

Children may be denied access to normal schools due to fear and stigmatization in the community (Human Rights Watch, 2004). For this reason, most children with HIV infection study in special schools. This study selected at random a sample of 70 children infected with HIV from Sneha Care Home and Shinning Star School, Bangalore, Karnataka. The children were undergoing treatment for HIV infection from Sneha Care Hospital, Bangalore. After checking medical reports, children with vertically transmitted virus (HIV-positive) were selected from Bangalore because Karnataka is one of the states in India that is worst affected by the spread of HIV with thousands of children infected with the virus. The control group included $70 \mathrm{HIV}$-negative participants at the same ages, selected at random from schools in Kerala, namely, Carmel Matha School and St Mary's School at Idukki. The normal cohorts (HIV-negative) were selected from Kerala due to time constraints and denial of permission for the study in normal schools at Bangalore. The two groups were matched for age, gender and ability in receptive and expressive communication in English. The groups only differed in their HIV status and any differences in the group in intellectual functioning on the WISC test could be attributed to their differing HIV status. Fear and discrimination in the society affect the quality of education in children with HIV infection. As a result, they were not encouraged to study in schools with normal cohorts. Alongside there is unavailability of teachers to educate these children. Hence, in most of these institutions, social workers take an initiative to educate the children. In most such institutions in Bangalore, children are provided fundamental education. These children do not follow any particular standard high school syllabus such as Indian Certificate of Secondary Education (ICSE), Central Board of 
Secondary Education (CBSE) or State level. The children with HIV infection were not divided into grades according to age, but rather were divided into batches based on their abilities and capabilities. The study chose samples from lower socio-economic status (SES) for both groups, due to the lack of availability of HIVinfected children from a middle SES. Evidence indicates that education typically available with middle/higher SES groups can decrease the risk of acquiring HIV (Goldman and Smith, 2002). Moreover, studies on young HIV groups of children are sparse. The study was designed to understand the difference in intellectual functioning between HIV-positive children and normal cohorts. Furthermore, possible interaction effects of gender, group and age on intellectual functioning were considered.

\section{Materials}

The WISC-IIIUK (Wechsler,1991) consisting of several subtests, each measuring a different facet of intelligence, was administered to each child. Some subtests require the child to reason abstractly, some focus on the child's memory, some on perceptual skills and so forth. No single subtest is intended to provide an adequate index of intelligent behaviour. Wechsler (1944) insisted that an intelligence scale should sample broadly from the full array of cognitive abilities and thus reflect the multi-faceted characteristics of intellectual ability. The materials used for each subtest are presented in Tables 1 and 2, respectively.

The above subtests yield three composite variables: the Verbal IQ (VIQ), Performance IQ (PIQ) and the Full-Scale IQ (FSIQ).

The factor index scores were used to calculate verbal comprehension (Verbal Comprehension Index (VCI)), perceptual organization (Perceptual Organization Index (POI)) and freedom from distractibility (Freedom from Distractibility Index (FDI)).

\section{Reliability}

The reliability of a test refers to its accuracy and consistency of measurement and to the stability
Table I. Subtests for Verbal and Performance IQ.

\begin{tabular}{ll}
\hline Verbal & Performance \\
\hline Information & Picture completion \\
Similarities & Coding \\
Arithmetic & Picture arrangement \\
Vocabulary & Object assembly \\
Comprehension & Block design \\
\hline
\end{tabular}

Table 2. Factor Index.

\begin{tabular}{lll}
\hline Factor-I & Factor-2 & Factor-3 \\
\hline Information & Picture completion & Arithmetic \\
Similarities & Picture arrangement & Digit span \\
Vocabulary & Block design & \\
Comprehension & Object assembly & \\
\hline
\end{tabular}

of its scores over time. The test reliabilities ranged from .80 to .97 for VIQ, PIQ, FSIQ and verbal comprehension. Reliability range for performance organization, processing speed and speed from distractibility indices was .56.89 , respectively. Inter-rater reliabilities for verbal subtests were more than .92 . The reliability coefficients for the WISC-III subtests, except coding and symbol search, were estimated by the split-half method. The items on each subtest were divided into two half tests that approximated parallel forms with approximately equal variances. Scores on the two half tests were then correlated and the resulting coefficient was corrected by the Spearman-Brown formula. For coding and symbol search subtests, stability coefficients were used as reliability estimates, because measures of internal consistency, such as split-half coefficients, are inappropriate for speeded tests. These stability coefficients were obtained from the test-retest study.

\section{Validity}

The manual reports strong correlations between WISC-III metrics and comparable metrics from the Wechsler Preschool and Primary Scale of Intelligence-Revised (WPPSI-R), Wechsler Intelligence Scale for Children-Revised (WISC-R), Wechsler Adult Intelligence Scale-Revised 
(WAIS-R), Otis-Lennon School Ability Test and Differential Ability Scales ( $r$ s between WISC-III IQs and comparable composites range from .59 to .92). Additionally, independent studies report correlations between WISC-III IQ/Indices and comparable metrics from other batteries that are well within acceptable limits. The WISC-III manual reports appropriate correlations with achievement. Studies published since test publication also report appropriate IQ achievement correlations in children representing normal, referred, learning disabled, severely emotionally disturbed, language/ speech impaired and hearing-impaired/deaf clinical categories.

\section{Procedure}

Tests were administered in a quiet room at school during a single, individual session. In order to avoid biasing of performance in any test from effects of practice or fatigue, the test presentation order was counterbalanced. The verbal and performance subtests were administered in alternating order to make the test session interesting and varied. If the child refused to respond to a particular subtest, it was administered later. In particular, children were presented with a total of 11 subtests: to assess VIQ, PIQ, FSIQ, verbal comprehension (VCI), perceptual organization (POI) and freedom from distractibility (FDI).

The VIQ is the ability to analyse information and solve problems using language-based reasoning and ability to deal with abstract social conventions, rules and expressions. The PIQ measures spatial perception, visual abstract processing, problem-solving abilities and the ability to quickly perceive visual details. The FSIQ assesses a child's ability to reason abstractly given both verbal and visual prompts. The VCI is an overall measure of verbal concept formation, the child's ability to verbally reason, and is also influenced by knowledge learned from the environment. POI measures nonverbal reasoning and inductive reasoning. FDI assesses the child's attention, concentration and working memory.

\section{Scoring procedures}

\section{Obtaining scaled scores, IQ scores and index scores}

After each WISC-III ${ }^{\mathrm{UK}}$ subtest was scored, each child's raw score and scaled subtest scores were obtained. The scaled score was used to calculate the VIQ, PIQ and FSIQ scores. The threefactor-based index scores were also calculated from the scaled scores. The raw score of each subtest was converted to scaled scores using the manual of WISC-IIIUK ${ }^{\mathrm{UK}}$ Scaled scores were based on the child's age in years, months and days.

The child's verbal score is the sum of scaled scores on the five regularly administered verbal subtests. The performance score is the sum of scaled scores on the five regularly administered performance subtests. The full scale score is the sum of the verbal and performance scores; hence, it is the sum of 10 subtest scaled scores. Similarly, each factor-based composite score is the sum of the scaled scores of its contributing subtests. Later, the verbal score, performance score and full scale scores were converted to their IQ equivalents. Similarly, the verbal comprehension score, perceptual organization/reasoning score and freedom from distractibility scores were converted to index scores.

\section{Results}

\section{Construction of IQ and index scores}

The sum of scaled scores (verbal, performance and full scale) were formed by summing each child's actual score on the relevant subtests and obtaining the corresponding scaled scores from the manual for scoring. For each of the seven age groups, the means and standard deviations (SDs) of the three sums of scaled score distributions were calculated. The construction of the three index scores (VCI, POI, FDI) followed the same general procedures. A review of data from age 6 to 12.11 years (both male and female) indicates higher scores for VIQ, PIQ, FSIQ, VCI, POI and FDI in normal children 
Table 3. Mean and SD of the IQ and index scores of the HIV-positive and HIV-negative (normal) children.

\begin{tabular}{|c|c|c|c|c|c|c|}
\hline & Female & Children & Male & Children & Total & Children \\
\hline & Mean & SD & Mean & SD & Mean & SD \\
\hline \multicolumn{7}{|l|}{ Verbal IQ } \\
\hline HIV +tve & 67.6 & 12.9 & 66.2 & 8.6 & 66.89 & 10.9 \\
\hline HIV -tve & 96 & 8.7 & 101.66 & 6.8 & 98.8 & 8.2 \\
\hline \multicolumn{7}{|l|}{ PIQ } \\
\hline HIV +tve & 56 & 6.8 & 56.6 & 6.6 & 56 & 6.7 \\
\hline HIV -tve & 76.8 & 4.5 & 76.4 & 5.5 & 76.6 & 5.4 \\
\hline \multicolumn{7}{|l|}{$\mathrm{FSI}$} \\
\hline HIV +tve & 58.8 & 9.9 & 59.1 & 8.6 & 59 & 9.2 \\
\hline HIV -tve & 86 & 6.1 & 88.3 & 5.2 & 87.8 & 5.8 \\
\hline \multicolumn{7}{|l|}{$\mathrm{VCl}$} \\
\hline HIV +tve & 65.9 & 12.3 & 65.6 & 8.2 & 65.7 & 10.4 \\
\hline HIV -tve & 98.2 & 5.6 & 103.2 & 4.1 & 100.7 & 5 \\
\hline \multicolumn{7}{|l|}{ POI } \\
\hline HIV +tve & 57.1 & 6.9 & 57.8 & 5.2 & 57.4 & 7 \\
\hline HIV -tve & 76.4 & 5.7 & 103.2 & 4.1 & 100.7 & 5 \\
\hline \multicolumn{7}{|l|}{ FDI } \\
\hline HIV +tve & 76.2 & 12.1 & 76.2 & 12.7 & 76.2 & 12.3 \\
\hline HIV -tve & 84.1 & 6.5 & 86.6 & 5.6 & 85.4 & 6.5 \\
\hline
\end{tabular}

SD: standard deviation; PIQ: Performance IQ; FSI: Full-Scale IQ; VCI: Verbal Comprehension Index; POI: Perceptual Organization Index; FDI: Freedom from Distractibility Index.

(HIV negative) as compared to children with HIV infection. The study used WISC-III norms for the assessment.

The results are presented according to the various IQ and index scores. The means and SDs of the HIV-infected and normal children are presented in Table 3. The means indicate that the normal HIV-negative children performed better than the HIV-positive children on all the subtests and have higher scores on the FSIQ than the HIV-positive children.

VIQ. Figure 1 shows the performance of the children on the VIQ test.

The average VIQ scores of children aged 6, $7,8,9,10,11$ and 12 years with HIV infection were 73.9, 66.1, 71.7, 63.6, 61.6, 69.2 and 62.1, respectively, whereas the corresponding figures for the normal cohorts were 97.6, 95, 97, 98.4, $99.1,97.3$ and 98.83 , respectively.

The means indicate that the HIV-positive children scored significantly lower than normal cohorts on all the verbal tests except arithmetic.

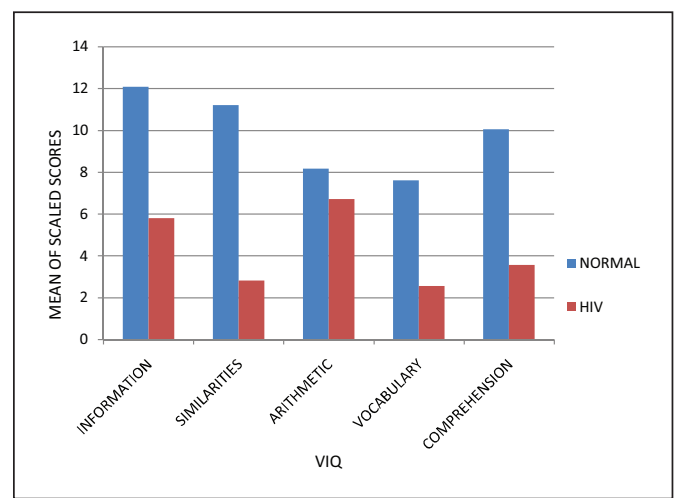

Figure I. Comparison of means of scaled scores in verbal intelligence quotient

Analysis of variance (ANOVA) computed with (2) group $\times(7)$ age $\times(2)$ gender indicates that the main effect of group was significant $(F(1,140)=321.171, p<.000)$ revealing that HIV-positive children scored less than HIVnegative children. The interaction effect of group and age was also significant $(F(6,140)=.993$, 


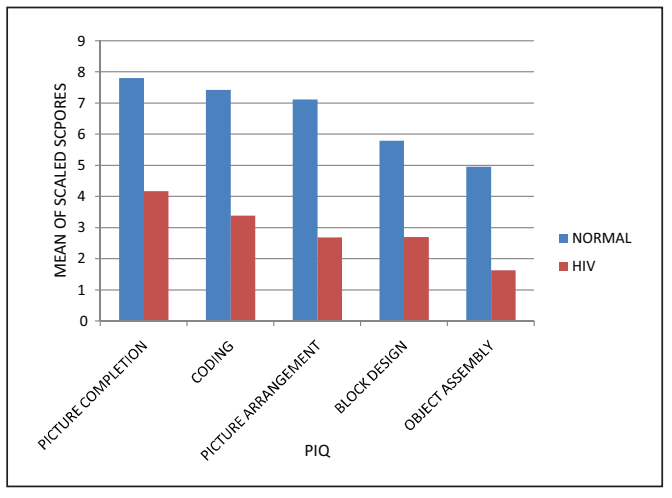

Figure 2. Comaprison of means of scaled scores in performance intelleigence quotient.

$p<.019)$. Post hoc Scheffe's test showed that the HIV-infected group has lower scores than the normal group at all ages $(p<.05)$.

PIQ.. Figure 2 shows the performance of the children on the PIQ test.

The average scores on PIQ for children aged $6,7,8,9,10,11$ and 12 years with HIV infection were $61.1,55.5,59,54.2,53,57.9$ and 53.4, respectively, whereas the corresponding figures for the normal cohorts were 84, 74.1, 79.4, 71.7, 76.9, 73.8 and 76.3, respectively.

The PIQ scores indicate that the HIVpositive children scored significantly lower than that of the normal children in all areas of the performance test.

ANOVA indicates that the main effect of group was significant $(F(1,140)=277.845$, $p<.000$ ) showing HIV-positive children scored less than HIV-negative children. The main effect of age was significant $(F(6,140)=4.168$, $p<.001$ ), indicating ages 6 and 9 years differed from the other age groups. The interaction of group, gender and age was also significant $(F(6$, $140)=2.404, p<.032$ ), indicating ages 6 and 9 years scored lower in the HIV group. Post hoc Scheffe's test showed that ages 6 and 9 years differ $(p<.05)$.

FSIQ score. The average scores on FSIQ for children aged $6,7,8,9,10,11$ and 12 years with HIV infection were 67.8, 57.8, 62.4, 55.8, 53.9,

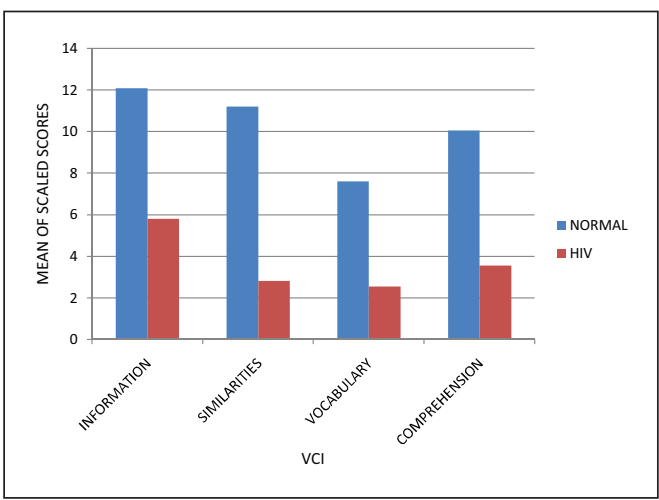

Figure 3. Comparison of means of scaled scores in test of verbal comprehension.

60.5 and 54.5, respectively, whereas the corresponding figures for the normal cohorts were $90.2,83,87.5,86.6,86.8,84.2$ and 91.9, respectively.

ANOVA indicates that the main effect of group was significant $(F(1,140)=320.867$, $p<.000)$ revealing HIV-positive children scored less than HIV-negative children. The main effect of age was significant $(F(6,140)=2.198$, $p<.048)$. Post hoc Scheffe's test showed that the HIV-infected group has lower scores than the normal group at all ages.

VCl. Figure 3 shows the performance of the children on the VCI.

The average scores on VCI for children aged $6,7,8,9,10,11$ and 12 years with HIV infection were 73.1, 64.7, 70.8, 63.8, 59.8, 67.9, 59.9 and 65.7 , respectively, whereas the corresponding figures for the normal cohorts were 102.9, 99.3, 101.5, 97.7, 97.8, 96.7 and 108.8, respectively. The means indicate that the HIV children scored much lower than that of the normal children in verbal comprehension.

ANOVA indicates that the main effect of group was significant $(F(1,140)=421.380$, $p<.000$ ), showing HIV-positive children scored less than HIV-negative children. The interaction of group and age was significant $(F(6$, $140)=2.361, p<.035)$. Post hoc Scheffe's test showed that the HIV-infected group has lower scores than the normal group at all ages $(p<.05)$. 


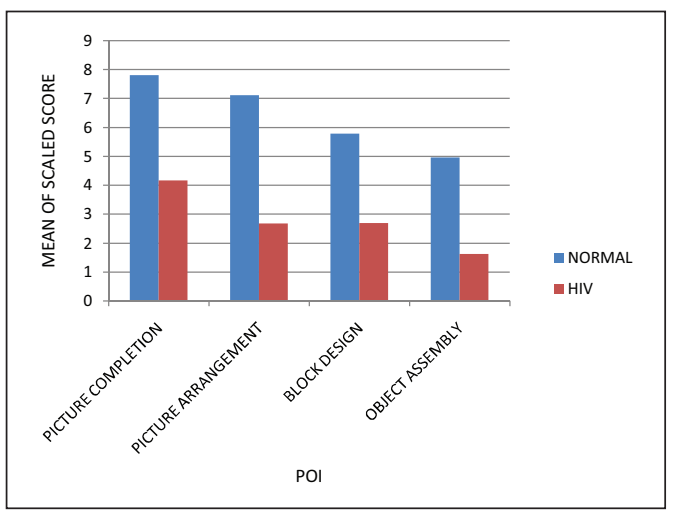

Figure 4. Comparison of mean of scaled scores in test of perceptual organization.

POI. Figure 4 shows the performance of the children on the POI.

The average scores on POI for children aged $6,7,8,9,10,11$ and 12 years with HIV infection were 63.8, 58.3, 58.2, 53.6, 52.9, 59.6 and 55.5 , respectively, whereas the corresponding figures for the normal cohorts were 88, 78.9, 77.2, 70.8, 74, 72.7 and 77.7, respectively.

The means show that the HIV-positive children scored less than that of normal children in the area of POI.

ANOVA indicates that the main effect of group was significant $(F(1,140)=206.902$, $p<.000$ ), showing that HIV-positive children scored less than HIV-negative children. The main effect of age was also significant $(F(6$, $140)=6.056, p<.000)$. Post hoc Scheffe's test showed that the HIV-infected group has lower scores than the normal group at all ages $(p<.05)$.

FDI. Figure 5 shows the performance of the children on the FDI.

The average scores on FDI for children aged $6,7,8,9,10,11$ and 12 years with HIV infection were $76.7,75.3,81.4,72.4,75.3,81.1$ and 71.2 , respectively, whereas the corresponding scores for the normal cohorts were 70.7, 75.3, $77.1,86.3,87.7,101.4$ and 99 , respectively.

The statistics show that the HIV-positive children scored lower than that of the normal children in the area of FDI.

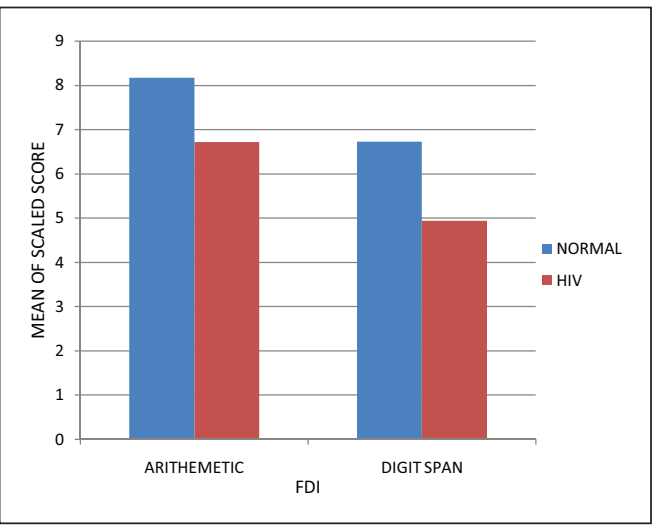

Figure 5. Comparison of means of scaled scores in test of freedom from distractibility.

ANOVA indicates that the main effect of group was significant $(F(1,140)=25.994$, $p<.000)$ showing HIV-positive children scored less than normal children. The main effect of age was significant $(F(6,140)=6.299, p<.000)$, indicating differences between ages 6,7 and 11 years. Post hoc Scheffe's test showed that the HIV children have lower scores than the normal at ages 6,7 and 11 years $(p<.05)$.

\section{Discussion}

To date, there has been a dearth of studies using WISC to assess children with HIV infection in India. The findings of this study indicate that HIV-positive children scored less in all the subtests of the WISC-IIIUK as compared to HIVnegative children. The results are discussed according to the factors in the test. Some of the factors that may play a vital role in the poor intellectual functioning of children with HIVpositive children are explained herewith. Stigma may prevent HIV-infected children from access to proper education, well-being, treatment and care both directly (through abuse, denial, loss of inheritance, etc.) and indirectly if the child avoids potentially stigmatizing situations such as social interaction or educational opportunities from internalizing stigma (Strode and Barrett-Grant, 2001). Second, women contact HIV through intravenous drug use or have 
partners with histories of drug use, and children with HIV are at increased risk of prenatal drug and alcohol exposure and premature birth, both of which have been associated with poor cognitive outcome (Henry et al., 1996; Loebstein and Koren, 1997). At the same time, maternal illness may compromise parenting (Kotchick et al., 1997), which, in turn, can negatively affect the child's intellectual functioning. But, to the best of our knowledge, there have been no studies reflecting a detailed statistical analysis on comparison of intellectual ability in this clinical and normal child population.

This study characterizes a detailed analysis of intellectual ability between HIV-positive children and normal cohorts, focussing mainly on the areas of VIQ, PIQ, FSIQ, VCI, POI and FDI. The descriptive statistics of VIQ portrays that the ability to analyse information, solve problems using language-based reasoning and ability to deal with abstract social conventions, rules and expressions are lower in HIV-positive school-age children when compared with normal cohorts. Studies that may compliment the findings are outlined. For example, Bisiacchi et al. (2000) found that on a series of memory tests, HIV-infected youth scored significantly lower than sero-reverters including a digit span task, but again, treatment regimens were not described. Language impairments were usually found in HIV-infected children, particularly in expressive language, the presence of both expressive and receptive language dysfunction in them suggests a more fundamental impairment (Moss et al., 1998).

Similarly, HIV-positive children scored less in PIQ when matched with HIV-negative cohorts, which, in turn, illustrates that the spatial perception, visual abstract processing, problem-solving abilities and the ability to quickly perceive visual details dwindle in HIV-positive children. The interpretation of FSIQ score reflects that the HIV-negative children have a higher IQ score when compared to HIV-positive children. Evidence revealed that failure to provide items such as toys geared to teaching about shapes and colours has been shown to be associated with reduced IQ scores in children (Bradley et al., 1989). The impact of deficits in neuronal growth can also be a causal factor.
Furthermore, detailed analyses of VCI exhibited that HIV-positive children scored less as compared to normal cohorts. The HIVpositive individuals, regardless of their stages of infection, reportedly have more frequent cognitive complaints than do HIV-negative individuals (Hinkin et al., 1998). Children with HIV infection scored lower in VCI because of their lack of ability to verbally reason which, in turn, may be a consequence of an unhealthy environment. Most of the HIV-infected children were staying in care homes. Several studies suggest that in some cases of extremely adverse rearing circumstances, well-functioning child care institutions may offer children a better rearing environment than their own dysfunctional families (e.g. Miller et al., 2007). But for healthy emotional, cognitive and even physical development of children, long-term institutional care is actually inappropriate for infants and young children because they require at least one consistent and loving caregiver with whom they can form a bond (United States Agency for International Development, 2004).

Moreover, the analysis of POI indicates that HIV-positive children scored less when compared with normal cohorts. This test measures nonverbal reasoning and inductive reasoning. HIV in children has long been associated with subtle and more specific cognitive impairments, including visuo-spatial and motor integration deficits (Fundaro et al., 1998). In WISC-III, freedom from distractibility measures concentration and attention of children. The results illustrate that the HIV-positive children scored below par in FDI when examined alongside the control group. Attention dysregulation is one among major problems faced by children with HIV infection (Brouwers et al., 1994; Lindsey et al., 2007). Analysis of FDI indicates that HIV-negative children have higher scores as compared to children with HIV infection. The causal factor for cognitive issues in HIVinfected children may be due to the effects of extreme poverty and lack of opportunity for stimulation during sensitive periods of development, prenatal drug exposure, malnutrition, family stress associated with death or illness of the primary caregiver, prematurity and other 
developmental disabilities unrelated to HIV (Coscia et al., 2001).

Neuropsychological impairments are more common, progress more quickly and may entail greater damage in children than in adults with HIV infection (Wachsler-Felder and Golden, 2002). However, since the introduction of combination therapies, children vertically infected with HIV have shown markedly improved immune function, appearing to exhibit global cognitive functioning comparable to their uninfected peers (Blanchette et al., 2002; Tardieu, 1998). Researchers had begun to evaluate the longitudinal efficacy of these new treatments on cognitive functioning of HIV-positive children (Gortmaker et al., 2001). Moreover, stigmatization and psychosocial distress experienced during childhood not only affect HIV-infected children's ability to capitalize on occupational and social opportunities for their own survival as adults but also have implications for their ability to contribute to national development (Sarada and Udamala, 2009).

In summary, the findings indicate that HIVpositive children scored less in intellectual functioning as compared to normal children of the same age. This investigation addresses general intellectual functioning, language functioning, spatial perception, visual abstract processing, problem-solving abilities, the ability to quickly perceive visual details, the child's ability to reason abstractly, verbal concept formation, nonverbal and inductive reasoning, concentration and working memory in HIVpositive children. This study helps future researches to identify the educational and vocational needs of children with HIV infection to bring about remedial and preventive processes.

By figuring out more precisely which cognitive ability or motor skills are most closely associated with disease progression, is helpful in making efficient use of time and resources. Since HIV-infected children live longer and develop normally like the normal children (Bisiacchi et al., 2000), reflecting on the multifaceted characteristics of intellectual ability in $\mathrm{HIV}$-infected children in the study provides an insight to the type of interventions that will assist HIV-infected children for successful academic careers. The study chose school-age children as they are in the formative stages of their lives, and therefore, understanding their intellectual abilities can assist their future development. A concentrated multidisciplinary approach in diagnosing and treating may enable the HIV-positive children to reach pinnacles of excellence.

\section{Acknowledgements}

The authors wish to acknowledge the cooperation of all the children and the staff of the different schools in Bangalore and Kerala, India.

\section{Funding}

This research received no specific grant from any funding agency in the public, commercial or notfor-profit sectors.

\section{References}

Bisiacchi PS, Suppiej A and Laverda A (2000) Neuropsychological evaluation of neurologically asymptomatic HIV infected children. Brain and Cognition 43: 49-52.

Blanchette N, Smith ML, King S, et al. (2002) Cognitive development in school-age children with vertically transmitted HIV infection. Developmental Neuropsychology 21: 223-241.

Bradley RH, Caldwell BM, Rock SL, et al. (1989) Home environment and cognitive development in the first three years of life: A collaborative study involving six sites and three ethnic groups in North America. Developmental Psychology 25: 217-235.

Brouwers P, Belman AL and Epstein L (1994) Central nervous system involvement: Manifestations, evaluation, and pathogenesis. In: Pizzo PA and Wilfert CM (eds) Pediatric AIDS: The Challenge of HIV Infection in Infants, Children and Adolescents (2nd edn). Baltimore, MD: Lippincott Williams \& Wilkins, pp. 433-455.

Brown LK, Lourie KJ and Pao M (2000) Children and adolescents living with HIV and AIDS: A review. Journal of Child Psychology and Psychiatry 41: 81-96.

Centers for Disease Control (1994) Revised guidelines for the performance of CD4+ T-cell determination in persons with human immunodeficiency 
virus (HIV) infection. Morbidity and Mortality Weekly Report 43(RR-3): 1-21.

Coscia JM, Christensen BK, Rolando RH, et al. (2001) Effect of home environment, socioeconomic status and health status on cognitive functioning in children with HIV-1 infection. Journal of Pediatric Psychology 26: 321-329.

Fassinou P, Elenga N, Rouet F, et al. (2004) Highly active anti-retroviral therapies among HIV-1 infected children in Abidjan, Cote d'Ivorie. AIDS 18: 1905-1913.

Fundaro C, Miccinesi N, Figliola N, et al. (1998) Cognitive impairment in school-age children with asymptomatic HIV infection. AIDS Patient Care and STDs 12: 135-140.

Goldman DP and Smith JP (2002) Can patient selfmanagement help explain the SES health gradient? Proceeding of the National Academy of Sciences of the United States of America 99: 10929-10934.

Gortmaker SL, Hughes M, Cervia J, et al. (2001) Effect of combination therapy including protease inhibitors on mortality among children and adolescents infected with HIV-1. New England Journal of Medicine 345: 1522-1528.

Greenfield RA, Rivera DM and Fryen RE (2013) Pediatric HIV infection - World Medical Library-Medscape. Available at: http://emedicine.medscape.com/article/965086-overview.

Henry RR, Christensen BK, Coscia JM, et al. (1996) Relationship between cognitive and immune functioning in children born to HIV-1 seropositive children. Developmental Neuropsychology 12: 283-298.

Hinkin CH, Castellon SA, Van Gorp WG, et al. (1998) Neuropsychological features of HIV disease. In: Van Gorp WG and Buckingham SL (eds) Practitioner's Guide to the Neuropsychiatry of HIV/AIDS. New York: The Guilford Press, pp. $1-14$.

Human Rights Watch (2004) Future Forsaken: Abuses against HIV/AIDS-Affected Children in India. New York: Human Rights Watch.

Kotchick BA, Brody GH, Forehand R, et al. (1997) The impact of maternal HIV infection on parenting in inner city, African-American families. Journal of Family Psychology 11: 447-461.

Lindsey JC, Malee KM, Brouwers P, et al. (2007) Neurodevelopmental functioning in HIVinfected infants and young children before and after the introduction of protease inhibitor based highly active antiretroviral therapy. Pediatrics 119: e681-e693.
Loebstein R and Koren G (1997) Pregnancy outcome and neurodevelopment of children exposed in utero to psychoactive drugs: The mother risk experience. Journal of Psychiatry \& Neuroscience 22: 192-196.

Mellins CA and Ehrhardt AA (1994) Families affected by pediatric acquired immune deficiency syndrome: Some sources of stress and coping. Journal of Developmental and Behavioral Pediatrics 15: S54-S60.

Millana-Cuevas LC, Portellano JA and MartinezArias R (2007) Neuropsychological impairment in human immunodeficiency virus positive children. Revista de Neurologia 44: 366-374.

Miller IW, Bishop DS and Herman DS (2007) Relationship quality among HIV patients and their caregivers. AIDS Care 19: 203-211.

Moss HA, Bose S, Wolters P, et al. (1998) A preliminary study of factors associated with psychological adjustments and disease control in school age children infected with the human immunodeficiency virus. Journal of Developmental and Behavioral Pediatrics 19: 18-25.

Nozyce M, Hittleman J, Muenz L, et al. (1994) Effect of perinatally acquired human immunodeficiency virus infection on neurodevelopment in children during the first two years of life. Pediatrics 94: 883-891.

Pearson DA, McGrath NM, Nozyce M, et al. (2000) Predicting HIV disease progression in children using measures of neuropsychological and neurological functioning. Pediatrics 106(6): e76.

Puthanakit T, Oberdorfer A, Akarthum M, et al. (2005) Efficacy of highly active antiretroviral therapy in HIV infected children participating in Thailands National Access to Antiretroviral Programme. Clinical Infectious Diseases 41: 100-107.

Sarada P and Udamala VM (2009) AIDS Education to College Students. New Delhi, India: Discovery Publishing.

Spira R, Lepage P, Msellati P, et al. (1999) Natural history of human immunodeficiency virus type 1 infection in children. A five year prospective study in Rwanda. Pediatrics 104(5): e56.

Strode A and Barrett-Grant K (2001) The Role of Stigma and Discrimination in Increasing Vulnerability of Children and Youth Infected With and Affected with HIV/AIDS. London: Save the Children.

Tardieu M (1998) HIV-1 and the developing central nervous system. Developmental Medicine and Child Neurology 40: 843-846. 
UNAIDS (2013) Report on the Global AIDS Epidemic.

UNAIDS (2014). The Global HIV/AIDS Epidemic. Available at: http://kff.org/global-health-policy/fact-sheet/the-global-hivaids-epidemic/

United States Agency for International Development. Population, Health and Nutrition Information Project (2004) Children on the Brink 2004: A Joint Report of New Orphan Estimates and a Framework for Action. Geneva, Switzerland: Joint United Nations Programme on HIV/AIDS. .

United States Agency for International Development (USAID) (2012) India's HIV Orphans and Vulnerable Children: Generating Evidence for Policy and Practice. Available at: http://www. bu.edu/cghd/files/2012/02/IndiaOVCreport100212-copy1.pdf

Wachsler-Felder JL and Golden CJ (2002) Neuropsychological consequence of HIV in children: A review of current literature. Clinical Psychology Review 22: 441-462.

Wechsler D (1944) The Measurement of Adult Intelligence (3rd edn). Baltimore, MD: Williams \& Wilkins.

Wechsler D (1991) The Wechsler Intelligence Scale for Children (3rd edn). San Antonio, TX: The Psychological Corporation.

WHO, UNAIDS, and United Nations Children's Fund (UNICEF) (2011) Global HIV/AIDS Response: Epidemic Update and Health Sector Progress Towards Universal Access, Progress report 2011. Geneva.

Wolters PL, Brouwers P, Moss HA, et al. (1995) Differential receptive and expressive language functioning of children with symptomatic HIV disease and relation to $\mathrm{CT}$ scan brain abnormalities. Pediatrics 95: 112-119. 